\title{
Pair Creation of Black Holes Joined by Cosmic Strings
}

\author{
R. Emparan* \\ Departamento de Física de la Materia Condensada Universidad del País Vasco, \\ Apartado 644, 48080 Bilbao, Spain
}

(Received 15 June 1995)

\begin{abstract}
We argue that production of charged black hole pairs joined by a cosmic string in the presence of a magnetic field can be analyzed using the Ernst metric. The effect of the cosmic string is to pull the black holes towards each other, opposing to the background field. An estimation of the production rate using the Euclidean action shows that the process is suppressed as compared to the formation of black holes without strings.
\end{abstract}

PACS numbers: 04.70.Dy, 11.27.+d, 98.80.Cq

The idea that some gravitational instantons can be interpreted as mediating tunneling processes leading to, e.g., spontaneous formation of black holes, is an attractive one, and has provided some hints on peculiarities of the yet-to-be-built quantum theory of gravity, such as topology changing processes and the statistical properties of black holes. The simplest example is provided by the Euclidean section of the Schwarzschild metric, which was interpreted in Ref. [1] as yielding the nucleation rate of black holes in a thermal bath. More recently there has been considerable interest in solutions that describe the spontaneous creation of black hole pairs. The instantons relevant here are obtained from the analytic continuation to Euclidean time of geometries related to the $C$-metric, whose Lorentzian section is known to represent a pair of black holes accelerating uniformly in opposite directions [2]. In general, these solutions possess conical singularities running along the axis in the direction joining the black holes, either between the black holes or running from each black hole to infinity. Physically, these singularities reflect the presence of forces acting on the black holes. As a general rule, a conical deficit tends to pull the black hole towards it, whereas a conical excess pushes in the opposite direction.

An interesting modification of the $C$-metric is the Ernst metric [3], obtained by adding a background electromagnetic field. This can be adjusted so as to compensate exactly for the necessary force to accelerate the black holes, and the resulting geometry is free from conical singularities. This solution has been extensively studied in recent years [4-8]. However, as we will argue below, this is not the only channel for the decay of the background magnetic field. In fact, one can consider processes where the black holes are joined by a cosmic string. It is then important to ascertain whether the presence of the string enhances or suppresses the probability for pair creation.

Below we will see that if, loosely speaking, the force exerted on the black hole by the background field is in excess to the product of its mass and acceleration then the Ernst metric has a conical deficit running in between the black holes. We will interpret this physically by regarding the conical deficit as created by a cosmic string which decelerates the black holes. [Very recently, another process involving similar ingredients but a different physical phenomenon (the breaking of a cosmic string to yield a pair of accelerating black holes) has been considered in Ref. [9]. It requires that the conical deficit runs in directions opposed to what we consider here.] Clearly, a question to consider is whether it is consistent to approximate a physical cosmic string by a conical singularity even in the vicinity of a black hole. This issue has been recently addressed in Ref. [10], where it is shown that a Nielsen-Olesen vortex can effectively pierce a black hole (and, in fact, it is only slightly distorted near the horizon) resulting in a geometry of a conical singularity centered on a black hole. Moreover, in the same paper it is argued that it is possible for a string to terminate on a black hole. This cannot be achieved in a topologically trivial space, since no gauge can be taken that remains regular as we shrink to zero radius a two-sphere connected to the string end. This topological obstruction disappears when the vortex terminates in a black hole, because the spheres cannot be contracted beyond the Schwarzschild radius. This opens up the exciting possibility of considering a new variety of string vortex-black hole interactions.

The black holes will be created in a background magnetic field, described by the Melvin solution [11]

$$
\begin{aligned}
d s^{2}= & \left(1+\frac{1}{4} \hat{B}_{M}^{2} \rho^{2}\right)^{2}\left(-d t^{2}+d z^{2}+d \rho^{2}\right) \\
& +\frac{\rho^{2} d \varphi^{2}}{\left(1+\frac{1}{4} \hat{B}_{M}^{2} \rho^{2}\right)^{2}}, \\
A_{\varphi}= & \frac{\rho^{2} \hat{B}_{M}}{2\left(1+\frac{1}{4} \hat{B}_{M}^{2} \rho^{2}\right)} .
\end{aligned}
$$

The motion of a pair of charged black holes uniformly accelerating in opposite directions in the background of a magnetic field is represented in Einstein-Maxwell theory 
by the Ernst solution:

$$
\begin{gathered}
d s^{2}=\frac{\Lambda^{2}}{A^{2}(x-y)^{2}}\left[G(y) d t^{2}-G^{-1}(y) d y^{2}+G^{-1}(x) d x^{2}\right]+\frac{G(x)}{\Lambda^{2} A^{2}(x-y)^{2}} d \varphi^{2}, \\
A_{\varphi}=-\frac{2}{B \Lambda}\left(1+\frac{1}{2} B q x\right)+k,
\end{gathered}
$$

where

$$
\begin{aligned}
\Lambda & =\left(1+\frac{1}{2} B q x\right)^{2}+\frac{B^{2}}{4 A^{2}(x-y)^{2}} G(x), \\
G(\xi) & =\left(1+r_{-} A \xi\right)\left(1-\xi^{2}-r_{+} A \xi^{3}\right),
\end{aligned}
$$

and $q^{2}=r_{-} r_{+}$. For a detailed description of this geometry see, e.g., Ref. [7]. Here we shall only summarize the most important features.

The parameters in Eq. (2) will be constrained so that $G(\xi)$ has four real roots $\xi_{1}<\xi_{2} \leq \xi_{3}<\xi_{4}$, and we will also set $\xi_{1}=-1 / r_{-} A$. Then, the correct signature is obtained when $x$ and $y$ are restricted to the ranges $\xi_{3} \leq x \leq \xi_{4},-\infty<y \leq x$. The surfaces $y=$ $\xi_{1}, \xi_{2}, \xi_{3}$ correspond to the inner black horizon, event black hole horizon, and acceleration horizon, respectively; $x=\xi_{3}, \xi_{4}$ are axes pointing towards spatial infinity and to the other black hole, respectively. It is not difficult to see that the parameter $A$ can loosely be thought of as the acceleration of the black holes. We will also define $m=\left(r_{+}+r_{-}\right) / 2$, which can be identified as the mass of the black hole. Another important feature is that the Ernst metric asymptotes to the Melvin metric at spatial infinity $\left(x, y \rightarrow \xi_{3}\right)$. Finally, $k$ will be taken so as to confine Dirac string singularities to the axis $x=\xi_{4}$.

It must be noted that $q$ and $B$ are not the physical magnetic charge and field, but rather they approximate them in the limit $r_{+} A, r_{-} A \ll 1$. In fact, the value of the magnetic field on the axis, where it takes its maximum value, is $\hat{B}=B G^{\prime}\left(\xi_{3}\right) / 2 L^{3 / 2}$ [we have defined $\left.L \equiv \Lambda\left(\xi_{3}\right)\right]$. Also, the adequate definition of the physical charge of the black hole is $\hat{q}=1 / 4 \pi \int F$, so that

$$
\hat{q}=q \frac{\Delta \varphi\left(\xi_{4}-\xi_{3}\right)}{4 \pi L^{1 / 2}\left(1+\frac{1}{2} q B \xi_{4}\right)},
$$

where $\Delta \varphi$ is the period of the azimuthal coordinate $\varphi$.

The semiclassical approximation is expected to be reliable for small values of $\hat{q} \hat{B}$. This will be equivalent to considering small $r_{+} A$. In this limit we find the following expressions for the roots $\xi_{i}$ :

$$
\begin{aligned}
& \xi_{2}=-\frac{1}{r_{+} A}+r_{+} A+\ldots, \\
& \xi_{3}=-1-\frac{r_{+} A}{2}+\ldots, \\
& \xi_{4}=1-\frac{r_{+} A}{2}+\ldots .
\end{aligned}
$$

Now, in general, the Ernst metric contains conical singularities running along the axes $x=\xi_{3}, \xi_{4}$. These can be avoided by properly adjusting the period of $\varphi$. The situation we are interested in requires the absence of conical singularities at the axis running to infinity. This is obtained by setting

$$
\Delta \varphi=\frac{4 \pi L^{2}}{G^{\prime}\left(\xi_{3}\right)} .
$$

If we also adjusted the value of the ratio circumference/radius at $x=\xi_{4}$ to match the value (6), we would obtain the regular metric considered in $[3,5,6,8]$. However, we have seen that it is also permissible to have a cosmic string joining the black holes and creating a conical deficit in between them. This requires

$$
G^{\prime}\left(\xi_{3}\right) \Lambda^{2}\left(\xi_{4}\right)>-G^{\prime}\left(\xi_{4}\right) L^{2} .
$$

In the limiting case (5), this inequality becomes approximately $q B>m A$, indicating that the effect of the string is to oppose to the force separating the black holes. The mass per unit length, $\mu$, of a cosmic string is obtained as $1 / 8 \pi$ times the conical deficit that it creates. Then, in our case,

$$
\mu=\frac{1}{4}\left(1-\left|\frac{G^{\prime}\left(\xi_{4}\right) L^{2}}{G^{\prime}\left(\xi_{3}\right) \Lambda^{2}\left(\xi_{4}\right)}\right|\right) \simeq q B-m A .
$$

With the choice (6) the physical magnetic field and charge in the weak field limit become

$$
\begin{aligned}
& \hat{B} \simeq B\left(1-\frac{1}{2} q B+2 \mu\right), \\
& \hat{q} \simeq q(1-2 \mu) .
\end{aligned}
$$

The procedure to obtain an instanton mediating the decay process of the magnetic field to a pair of black holes joined by a string has been described in Ref. [5]. First, we continue $t=i \tau$ in the metric (2). Positive definiteness of the metric then requires $\xi_{2} \leq y \leq \xi_{3}$. For nonextremal black holes, with $\xi_{1} \neq \xi_{2}$, regularity at the horizons requires adjusting the surface gravities and the period of $\tau$ (i.e., the inverse temperature $\beta$ ) so that

$$
\beta=\frac{4 \pi}{G^{\prime}\left(\xi_{3}\right)}=-\frac{4 \pi}{G^{\prime}\left(\xi_{2}\right)} .
$$

The second equality is achieved when $\xi_{1}-\xi_{2}-\xi_{3}+$ $\xi_{4}=0$, which imposes the following restriction on the parameters:

$$
A=\frac{r_{+}-r_{-}}{2 q^{2}} \sqrt{\frac{m}{r_{-}}} .
$$

In the limit of small $r_{+} A$, this means that the black holes are close to extremality. Equations (8),(9),(11) can be 
used to express all the parameters in terms of $\hat{q}, \hat{B}$, and $\mu$ :

$$
\begin{aligned}
r_{ \pm} & \simeq \hat{q}[1+2 \mu \pm(\hat{q} \hat{B}-\mu)], \\
m & \simeq q \simeq \hat{q}(1+2 \mu) \\
A & \simeq \hat{B}\left[1+\frac{1}{2} \hat{q} \hat{B}-\frac{\mu}{\hat{q} \hat{B}}(1+2 \hat{q} \hat{B})\right] .
\end{aligned}
$$

Notice that the effect of $\mu$ is to decrease the value of $A$, so the string effectively decelerates the motion of the black holes. It is also amusing to find that if we define, as in Ref. [10], the black hole internal energy (or inertial mass) by $m_{I} \simeq m(1-2 \mu)$ (we are taking into account that only half of the string is pinched on the black hole), then $m_{I}=\hat{q}$.

For extremal black holes, the event horizon is at infinite proper distance and we need only match $\beta$ at the acceleration horizon $y=\xi_{3}$.

Now we can slice the Euclidean solution in half along a constant $\tau$ surface (we have to take two antipodal values of $\tau$ ). The resulting geometry is precisely that of the moment of closest approach of the black holes in the Lorentzian-Ernst metric. Moreover, since the extrinsic curvature vanishes for both surfaces, they can be glued together. The process described in this way is the quantum tunneling from the Melvin metric to a pair of static black holes joined by a string that subsequently accelerate to infinity. If semiclassical reasoning is valid, then the rate of the process in first approximation should be given by $\exp \left(-I_{\mathrm{cl}}\right)$, with $I_{\mathrm{cl}}$ the action of the whole Euclidean solution. One could worry about the issue of the finiteness of the quantum corrections to the decay rate. This is very hard to analyze in a metric with not a very high degree of symmetry, like Eq. (2). Difficulties could be expected due to the infinite redshift in the presence of horizons. However, the qualitative aspects of this problem can be thoroughly analyzed in the much simpler case of thermal nucleation of black holes using the Schwarzschild instanton [1], and in this case it has been shown [12] that infinities can be properly renormalized in a low energy expansion of the Einstein-Hilbert action.

The calculation of the Euclidean action can be performed along the same lines as that for a pair of black holes without a string. In fact, one can see that the derivation given in Ref. [8] (see also Refs. [6,13]) carries over directly, so we will only quote the final result:

$$
I_{\mathrm{cl}}=\frac{2 \pi L^{2}}{G^{\prime}\left(\xi_{3}\right) A^{2}\left(\xi_{3}-\xi_{1}\right)} .
$$

This result is valid for both the extremal and nonextremal cases.

Now we must express the action in terms of the physical parameters, which we do in the weak field limit:

$$
I_{\mathrm{cl}}=\pi \hat{q}^{2}\left[\frac{1}{\hat{\hat{q}} \hat{B}}-\frac{1}{2}+\frac{\mu}{\hat{q} \hat{B}}\left(\frac{1}{\hat{q} \hat{B}}+2\right)+\cdots\right],
$$

for the nonextremal case. The result for the extremal case can be obtained by subtracting the black hole area contribution, $-\mathcal{A}_{\mathrm{bh}} / 4 \simeq-\pi \hat{q}^{2}$ from Eq. (14) [8].

We see in Eq. (14) that the action is increased when there is a non-null string energy density $\mu$. Therefore creation of black holes joined by a cosmic string is suppressed relative to the case where no string is present. In fact, we can give a simple heuristic derivation of the contribution of the string to the action. If we consider a particle of mass $\delta m$ at temperature $T$, its action is given by $\delta I=\delta m / T$. Regard now the string as a collection of particles of mass $\mu \times$ (proper length), i.e., $\delta m=$ $\mu \sqrt{\left|g_{y y}\right|_{x}=\xi_{4}} \delta y$ at a local inverse temperature $T_{y}^{-1}=$ $\beta{\sqrt{\left|g_{t t}\right|}}_{x=\xi_{4}}$. Then we calculate the string the action as

$$
\begin{aligned}
I_{\text {string }} & =\mu \int_{\xi_{2}}^{\xi_{3}} d y \beta \sqrt{\left|g_{t t} g_{y y}\right|_{x}=\xi_{4}} \\
& \simeq \frac{\pi \mu}{\hat{B}^{2}}
\end{aligned}
$$

which correctly reproduces the leading contribution of the cosmic string to Eq. (14). Of course, $I_{\text {string }}$ is nothing but the Nambu-Goto action of the string (the fact that vortices in the Abelian Higgs model are effectively described by the Nambu-Goto action was arrived at in Ref. [14]). Therefore we arrive at the simple result that the black hole pair production rate is modified in the semiclassical approximation just by the nucleation rate of the cosmic string.

We have been considering that the black holes are pulled apart by an electromagnetic field. Another force capable of accelerating black holes is inflation. This requires considering the cosmological $C$-metric, which has been studied in Ref. [15]. The results qualitatively resemble those for the Ernst metric, so we can be quite certain that the results presented here carry over to the cosmological case without much difficulty.

We are indebted to Ana Achúcarro and Juan Luis Mañes for useful conversations and encouragement. This work has been partially supported by a FPI grant from MEC (Spain) and projects UPV 063.310-EB119-92 and CICYT AEN93-0435.

*Electronic address: wmbemgar@lg.ehu.es

[1] D. J. Gross, M. J. Perry, and L. G. Yaffe, Phys. Rev. D 25 , 330 (1982).

[2] W. Kinnersley and M. Walker, Phys. Rev. D 2, 1359 (1970).

[3] F. J. Ernst, J. Math. Phys. 17, 515 (1976).

[4] G. W. Gibbons, in Proceedings of 22nd Karpacz Winter School of Theoretical Physics: Fields and Geometry, Karpacz, Poland, 1986, edited by A. Jadczyk (World Scientific, Singapore, 1986).

[5] D. Garfinkle and A. Strominger, Phys. Lett. B 256, 146 (1991). 
[6] D. Garfinkle, S. B. Giddings, and A. Strominger, Phys. Rev. D 49, 958 (1994).

[7] H.F. Dowker, J.P. Gauntlett, S. B. Giddings, and G. T. Horowitz, Phys. Rev. D 50, 2662 (1994).

[8] S. W. Hawking, G. T. Horowitz, and S. F. Ross, Phys. Rev. D 51, 4302 (1995).

[9] S. W. Hawking and S.F. Ross, preceding Letter, Phys. Rev. Lett. 75, 3382 (1995).

[10] A. Achúcarro, R. Gregory, and K. Kuijken, Report No. DTP/95/21, gr-qc/9505039.
[11] M. A. Melvin, Phys. Lett. 8, 65 (1964).

[12] J. L.F. Barbón and R. Emparan, Report No. PUPT-1529, EHU-FT 95/5, hep-th/9502155.

[13] S.W. Hawking and G.T. Horowitz, Report No. DAMTP/R 94-52, UCSBTH-94-37, gr-qc/9501014.

[14] D. Förster, Nucl. Phys. B81, 84 (1984); R. Gregory, Phys. Lett. B 206, 199 (1988).

[15] R. B. Mann and S.F. Ross, Report No. DAMTP/R-95/9, gr-qc/9504015. 